\title{
La identidad del educador infantil desde las narrativas biográficas
}

The Identity of the Early Childhood Educator from Biographical Narratives

Artículo de investigación | Research article

Fecha de recepción: 15 de junio de 2020

Fecha de aceptación: 09 de marzo de 2021 Fecha de disponibilidad en línea: octubre de 2021

doi: 10.11144/Javeriana.m14.iein

María Ángeles Hernández-Prados mangeles@um.es

UnIVERSIDAD DE MURCIA, ESPAÑA (D) ORCID: https://orcid.org/0000-0002-3617-215X

Amalia Ayala De la Peña amayala@um.es

UnIVERSIDAD de MURCIA, ESPAÑA (D) ORCID: https://orcid.org/000-0002-5585-9600

Para citar este artículo | To cite this article Hernández-Prados, M. Á. \& Ayala De la Peña, A. (2021). La identidad del educador infantil desde las narrativas biográficas. magis, Revista Internacional de Investigación en Educación, 14, 1-23. doi: 10.11144/Javeriana.m14.iein 


\title{
Resumen
}

El trabajo presenta el análisis de 123 entrevistas semiestructuradas realizadas a profesionales de educación infantil de la región de Murcia (España), durante el curso 2019/2020, con la finalidad de identificar los factores que inciden en la construcción identitaria del docente. El contenido se trató de forma sistemática, cualitativa y cuantitativamente, siguiendo las fases de Cisterna (2005), midiendo la frecuencia de aparición de las categorías establecidas mediante triangulación. Los resultados muestran la relevancia de las experiencias de la infancia-adolescencia, la docencia con los alumnos, la formación permanente y la capacidad de adaptabilidad, en contraposición al paso por la universidad y los aspectos normativos.

\section{Palabras clave}

Entrevista; educación de la primera infancia; enseñanza superior; biografía; cualificación del docente; centro de formación de profesorado; docente de preescolar

\author{
Abstract \\ The research presents the analysis of 123 semi-structured \\ interviews conducted with Early Childhood Educators in the \\ Region of Murcia (Spain) during the 2019/2020 academic \\ year in order to identify the factors that affected the identity \\ construction of the teacher. The analysis followed systematic, \\ qualitative and quantitative methodologies, based on the \\ phases of Cisterna (2005), which measured the frequency of \\ appearance of the categories established by triangulation. \\ Results showed the relevance of childhood-adolescent \\ experiences, teaching with students, permanent professional \\ development and adaptability capacity, as opposed to passing \\ through university and its regulatory aspects
}

\section{Keywords}

Interviews; early childhood education; higher education; biographies; teacher qualifications; teacher education school; preschool teachers 
Descripción del artículo | Article description

Este artículo de investigación, derivado del Proyecto de innovación en el Grado de Educación Infantil en la asignatura 1989 Fundamentos Teóricos de la Educación Infantil, es fruto de más de una década de trabajo como profesores en el grado de educación infantil, así como de diversos proyectos de innovación, en los que se aborda el tema de la construcción de la identidad docente.

\section{Identidad profesional docente y narrativa biográfica}

La construcción de una cultura escolar más democrática, en la que los profesionales, "frente a la imagen burocrática e impersonal del oficio docente, se quieren inscribir en un nuevo profesionalismo, donde se recupera la 'autor-idad' sobre su propia práctica, y el sujeto se expresa como 'autor' de los relatos de prácticas" (Bolívar, 2014, p. 713), ha evolucionado, en el marco de la labor docente, hacia la construcción de un profesional más dialógico, reflexivo e inclusivo (Saiz-Linares et al., 2019). Ahora bien, en la consideración de las voces docentes, la dimensión infantil tiene sus propias particularidades. El interés que despierta esta profesión no es equiparable, históricamente, con el que se muestra en otros niveles (educación primaria, secundaria y superior). El carácter asistencial de su origen, su no obligatoriedad e incorporación tardía al sistema formal de educación, así como la diversidad de términos empleados para referirse a ella, han contribuido al cuestionamiento de su relevancia.

Sin embargo, conscientes de la importancia de los primeros años de vida, y fruto de la evolución experimentada por las sociedades europeas en general, y por las familias en particular, la educación infantil "ha pasado a realizar un importante papel de apoyo a las familias, yendo más allá de una 
mera función asistencial, pretende llevar a cabo una esencial misión educativa" (Llorent, 2013, p. 29). El impacto del docente infantil sobre los futuros ciudadanos responsables del mundo otorga relevancia y responsabilidad a su labor y a las competencias necesarias para su desempeño. De modo que su perfil debe caracterizarse por una alta motivación, sentimiento de pertenencia, compromiso con los contextos de exclusión y pobreza (PérezAbril et al., 2014), y estar más inclinado a habilidades afectivo-emocionales que curriculares (Carbonero et al., 2012). Además, debe tener una personalidad crítica, sensible, proactiva y comprometida; disponer de formación y recursos pedagógicos amplios; poseer habilidades comunicativas y sociales; estar en capacidad de formar integralmente y desempeñar sus tareas con talento y con altos niveles de calidad (Carrasco et al., 2012).

El interés sobre cómo se construye la identidad docente y cómo esta influye en la práctica y en los contextos escolares es una temática con diversas aristas de estudio, que cuenta con un elevado nivel de investigación (Gajardo-Asbún, 2019). Uno de los ejes principales de investigación sobre la identidad docente es el de la formación inicial universitaria, pues las competencias adquiridas durante las practicas escolares que aparecen relacionadas con las técnicas y estrategias de evaluación son las que, según los futuros docentes de infantil, inciden significativamente en su identidad como docentes (González et al., 2019).

Aunque existen diversos modelos teóricos sobre la identidad docente, fruto de una concepción multidimensional y dinámica, que se inicia generalmente con la formación inicial y que abarca la socialización en los escenarios reales de aprendizaje y la participación en redes colaborativas que contribuyen a la construcción de un perfil profesional más reflexivo (González et al., 2019), desde una perspectiva amplia e integradora hemos adoptado un modelo que comienza incluso antes de empezar la vida universitaria (Sarceda, 2017). Abordar la identidad del educador infantil implica entender su construcción como un proceso continuo, que se nutre de elementos que el propio maestro considera relevantes y significativos en el ejercicio de dar sentido a su hacer profesional a lo largo del tiempo y con referentes variados. Los factores personales y biográficos, las relaciones con la comunidad educativa, el contexto socioeducativo y las experiencias en el aula (Norman, 2020), además de aspectos como el origen y desarrollo del docente, la imagen profesional, el carácter temporal, el reconocimiento social (Balderas, 2013) y la contextualización, entendida en el sentido de que "los profesores no son una mano de obra intercambiable de un país a otro, porque una buena parte de su trabajo se comprende en estricta relación con el enraizamiento histórico y sociocultural de la escuela de cada país" (Cantón y Tardif, 2018, p, 9), son una muestra de la complejidad en la 
que nos movemos. Los hitos relevantes en la construcción de la identidad docente, señalados por la investigación, son múltiples y, mayoritariamente, derivan de métodos cualitativos (Cantón et al., 2017; Carbonero et al., 2012; Norman, 2020; Sarceda, 2017), omitiendo el tratamiento cuantitativo de esta temática.

Tomar en consideración los sistemas sociales y escolares nos lleva a constatar el interés creciente, tanto académico como político, de la educación infantil en España en las dos últimas décadas. Sin duda, las directrices tanto de la Unión Europea como de la OECD (2017), con sus sucesivas ediciones de Starting Strong, inciden en la proliferación de análisis generales de políticas nacionales, tendencias y agendas comunes de implantación de medidas, siempre precedidas de estados de la cuestión o mapas de cifras clave, como recientemente ha ocurrido con lo relativo a la educación y atención a la primera infancia en Europa (Eurydice, 2019). Según el último informe del Ministerio de Educación y Formación Profesional de España (2019), las diferencias significativas entre población atendida, en función de comunidades autónomas, no es óbice para la prácticamente generalizada implantación (97.4 \%) de dichas directrices, lo que nos sitúa por encima de la media europea y 2.4 puntos por encima de ese objetivo específico para 2020. Para el caso de la región de Murcia, que presenta uno de los índices de pobreza más altos de España, con presencia superior de alumnado de procedencia extranjera, los retos no son muy distintos de los que afrontan el resto de niveles educativos.

Atendiendo a la revisión bibliográfica, en nuestro estudio nos hemos acercado a la idea de identidad desde un punto de vista sociocultural. Consideramos que la construcción de la identidad docente es un proceso continuo, en el que las experiencias vitales que dejan huella pueden ser múltiples y variadas, además de que no se dan en el vacío, sino en un contexto concreto. Con base en ello, nos planteamos la siguiente cuestión como problema de investigación: ¿Cómo evoluciona el desarrollo de la identidad docente y qué elementos son los que determinan, en mayor medida, la construcción de dicha identidad en el educador infantil? Para delimitar la línea temporal, así como los aspectos conceptuales-categóricos apriorísticos, se ha seguido el modelo establecido por Sarceda (2017). De este modo, el objetivo de la investigación consiste en identificar los factores señalados con más frecuencia por los docentes del área educación infantil en sus narrativas biográficas, respecto a los hitos vitales que inciden en la construcción de su identidad profesional, según las siguientes etapas: niñez y adolescencia, toma de decisiones, paso por la universidad, años de docencia y años de madurez. 


\section{Metodología}

El abandono de planteamientos dicotómicos, en pro de la confluencia y complementariedad de los métodos cuantitativos y cualitativos, supone una opción metodológica en alza. De los seis tipos de diseño de la investigación con método mixto de Pereira (2011), hemos optado por una investigación exploratoria secuencial o por etapas, con datos cualitativos recabados mediante un enfoque narrativo, en la primera etapa, y análisis estadístico cuantitativo, en la segunda; esta última es la que mostraremos en este estudio. Siguiendo las fases de Cisterna (2005), tras la recogida de las historias de vida, se procede a la delimitación conceptual y operacional de las categorías y subcategorías apriorísticas, y al procesamiento de la información desde la triangulación hermenéutica que posibilita el análisis cuantitativo de la información. A partir de la consideración del ámbito subjetivo de la experiencia humana, se llevó a cabo este estudio con el objetivo de identificar los elementos más significativos y frecuentes en la construcción de la identidad como docentes del nivel de educación infantil. Todo ello aportará claridad a los procesos de construcción de la identidad profesional en estos docentes y permitirá incidir desde la formación inicial universitaria en los factores más relevantes.

\section{Participantes}

En este estudio participaron 123 docentes de educación infantil de la Comunidad Autónoma de Murcia (España) durante el curso 2019/2020; 95.1 \% de ellos mujeres. La mayoría de los docentes ejercían su labor educativa en el momento de la entrevista, aunque también se registraron algunos casos de profesores en estado de jubilación. La edad mínima registrada es 25 años y la máxima 80; siendo el promedio de 46.72 años. Se observa que la distribución de los docentes, según la edad, es menor en los extremos, es decir, el $9 \%$ tiene menos de 30 años y el $9.9 \%$ es mayor de 60, mientras que en edades intermedias la presencia es la siguiente: $22.6 \%$, entre 30 y 40 años; $26.8 \%$, entre $41-50$ años, y el $31.7 \%$, entre 51 y 60 años.

\section{Instrumento y procedimiento de recogida de información}

De la diversidad de instrumentos que se han consultado para abordar la identidad docente (Balderas, 2013; Cantón et al., 2017), se ha seleccionado la entrevista semiestructurada de Sarceda (2017), porque es una de las más completas, pues indaga en toda la historia vital de la persona, no solo en el periodo de actuación profesional, y porque está centrada en el área infantil. El protocolo guía de la entrevista se encuentra conformado 
por 32 núcleos de cuestiones distribuidos en cinco dimensiones, correspondientes a los momentos clave del recorrido vital de los profesionales: 1) infancia-adolescencia; 2) toma de decisiones; 3) paso por la universidad; 4) años de docencia; y 5) años de madurez. A partir de las preguntas realizadas, los entrevistados reflexionaron y contaron las experiencias vitales más significativas que influyeron en su decisión de convertirse en docentes, así como su percepción sobre el ejercicio de la profesión, además de que señalaron los aspectos positivos y negativos que se han encontrado en el itinerario individualizado de vivencias acumuladas durante sus años de docencia.

Para la recopilación de datos, se empleó la entrevista semiestructurada mediante la técnica conversacional, permitiendo que el orden, formulación y cantidad de cuestiones pudiera ser alterado libremente en la interacción personal entre investigador y entrevistado. Aunque se dispone de una guía de cuestiones que responden a los objetivos de la investigación, estas pueden modificarse en función de cada sujeto entrevistado y de los intereses del investigador (Blasco-Hernández \& Otero-García, 2008; Hurtado, 2010).

La mayoría de las entrevistas se realizaron presencialmente en los centros educativos, y otras telefónicamente, por un grupo de maestros de formación del grado de educación infantil, familiarizados y entrenados mediante simulacros en el instrumento de recogida de información, mientras que el proceso de supervisión y toma de decisiones respecto de la pertinencia y adecuación de la entrevista al estudio, así como el análisis de los datos, fue realizado por el equipo de investigación de forma colaborativa. Las entrevistas tienen una duración aproximada de 30 minutos, siendo grabadas en audio con autorización de los entrevistados, según un acuerdo de confidencialidad sobre el manejo de la información recogida, y luego fueron transcritas para facilitar el análisis de contenido.

\section{Análisis de contenido}

Tras la delimitación del tipo de investigación y del diseño metodológico, y atendiendo al ámbito temático, el problema de investigación, la revisión bibliográfica y a las cuestiones que se contemplan en el protocolo guía de la entrevista semiestructurada, se establecieron las categorías y subcategorías apriorísticas (tabla 1), para tener mayor facilidad en la sistematización y objetividad en el análisis del contenido, además de facilitar la identificación de las unidades de texto segmentadas y la interpretación de las mismas, mediante la triangulación hermenéutica (Cisterna, 2005). La entrevista favorece la segmentación del texto en cinco dimensiones o categorías principales y, posteriormente, en treinta subcategorías. No obstante, 
el análisis de contenido realizado por el equipo de investigación, sobre una selección hecha al azar de diez entrevistas, amplió el volumen de subcategorías, además de que permitió la tipificación y codificación del contenido en cada una de ellas, facilitando así la sistematización de la información en Excel y, tras su depuración, su ingreso en el software estadístico SPSS.

Tabla 1

Tipificación y codificación de las categorías y subcategorías apriorísticas de la identidad docente en educación infantil

\begin{tabular}{|c|c|c|c|}
\hline Categorías & Subcategorías & Tipificación & Codificación \\
\hline \multirow{16}{*}{$\begin{array}{l}\text { Infancia- } \\
\text { Adolescencia }\end{array}$} & \multirow{5}{*}{$\begin{array}{l}\text { A1. Datos } \\
\text { personales }\end{array}$} & A1. Género & 1. Hombre; 2. Mujer \\
\hline & & A1. Edad & Numérico \\
\hline & & A1. Murcia & 1. Sí; 2. No \\
\hline & & A1. Municipio Murcia & 1. Sí; 2. No \\
\hline & & A1. Otras comunidades & Nominal - Cadena \\
\hline & \multirow{3}{*}{$\begin{array}{l}\text { A2. Situación } \\
\text { familiar }\end{array}$} & A2. Tipología familiar & $\begin{array}{l}\text { 1. Nuclear; 2. Extensa; } \\
\text { 3. Monoparental; 4. Otras }\end{array}$ \\
\hline & & A2. Miembros familiares & Numérico \\
\hline & & A2. No hijos & Numérico \\
\hline & A3. Educación & A3. Educación & Nominal-Cadena \\
\hline & \multirow{5}{*}{ A4. Escolaridad } & A4. Escolaridad & Nominal-Cadena \\
\hline & & A4. Relación profesorado & Nominal-Cadena \\
\hline & & A4. Relación compañeros & Nominal-Cadena \\
\hline & & \multirow[t]{2}{*}{ A4. Relación familia-escuela } & 1. Sí; 2. No \\
\hline & & & Nominal-Cadena \\
\hline & \multirow[t]{2}{*}{ A5. Otros } & \multirow[t]{2}{*}{ A5. Aspectos de la infancia } & 1. Sí; 2. No \\
\hline & & & Nominal-Cadena \\
\hline \multirow{4}{*}{$\begin{array}{l}\text { Toma de } \\
\text { decisiones }\end{array}$} & B1. Cuando & B1. Momento de la decisión & 1. Infancia; 2. Adolescencia; 3. Madurez \\
\hline & \multirow[t]{2}{*}{ B2. Autonomía } & B2. Autonomía & 1. Sí; 2. No \\
\hline & & B2. Referentes-apoyo & $\begin{array}{l}\text { 1. Familia; 2. Profesorado; } \\
\text { 3. Amigos; 4. Cambio de carrera; } \\
\text { 5. Mercado laboral }\end{array}$ \\
\hline & B3. Titulación & B3. Fecha de inicio & Numérico y posteriormente por intervalos \\
\hline \multirow{8}{*}{$\begin{array}{l}\text { Paso por la } \\
\text { universidad }\end{array}$} & C1. Magisterio & C1. Estudios de Ed. Infantil & Nominal-Cadena \\
\hline & \multirow[t]{2}{*}{ C2. Docente } & C2. Transmisor de conoc. & 1. Sí; 2. No \\
\hline & & C2. Docente cercano & 1. Sí; 2. No \\
\hline & C3. Modelo & C3. Influyó como docente & 1. Sí; 2. No \\
\hline & C4. Politizados & C4. Ideologizados & 1. Sí; 2. No \\
\hline & \multirow{3}{*}{ C5. Aplicabilidad } & C5. Transferencia & 1. Sí; 2. No \\
\hline & & C5. Nivel de transferencia & $\begin{array}{l}\text { 1. Nada; 2. Poco; 3. Algo; } \\
\text { 4. Bastante; 5. Mucho }\end{array}$ \\
\hline & & C5. Aspectos & Nominal-Cadena \\
\hline
\end{tabular}




\begin{tabular}{|c|c|c|c|}
\hline \multirow{15}{*}{$\begin{array}{l}\text { Años de } \\
\text { docencia }\end{array}$} & \multirow{2}{*}{$\begin{array}{l}\text { D1. Reconocim. } \\
\text { docente }\end{array}$} & D1. Reconocido & 1. Sí; 2. No \\
\hline & & D1. Agente & $\begin{array}{l}\text { 1. Familia; 2. Docentes; } \\
\text { 3. Sociedad; 4. Niños }\end{array}$ \\
\hline & D2. Aislamiento & $\begin{array}{l}\text { D2. Aislado } \\
\text { profesionalmente }\end{array}$ & 1. Sí; 2. No \\
\hline & D3. Docentes & D3. Relación con docentes & Nominal-Cadena \\
\hline & \multirow[t]{2}{*}{ D4. Alumnado } & D4. Experiencia positiva & Nominal-Cadena \\
\hline & & D4. Experiencia negativa & Nominal-Cadena \\
\hline & \multirow{4}{*}{$\begin{array}{l}\text { D5. Educación } \\
\text { emocional }\end{array}$} & D5. Estabilidad emocional & Nominal-Cadena \\
\hline & & D5. Influencia en el aprend. & 1. Sí; 2. No \\
\hline & & $\begin{array}{l}\text { D5. Importancia del } \\
\text { contexto }\end{array}$ & 1. Sí; 2. No \\
\hline & & D5. Tipo de contexto & Nominal-Cadena \\
\hline & D6. Efectos & D6. Previsión de los efectos & 1. Sí; 2. No \\
\hline & D7. Cambios & D7. Evolución docente & 1. Sí; 2. No \\
\hline & $\begin{array}{l}\text { D8. } \\
\text { Infravalorado }\end{array}$ & D8. Infravalorado & 1. Sí; 2. No \\
\hline & D9. Centro & D9. Afecta al centro & 1. Sí; 2. No \\
\hline & D10. Género & D10. Diferencias de género & 1. Sí; 2. No \\
\hline \multirow{8}{*}{$\begin{array}{l}\text { Años de } \\
\text { madurez }\end{array}$} & \multirow{2}{*}{$\begin{array}{l}\text { E1. Aspectos } \\
\text { normativos }\end{array}$} & E1. Cambios de ley & 1. Sí; 2. No \\
\hline & & E1. Valoración de reformas & Nominal-Cadena \\
\hline & \multirow[t]{2}{*}{ E2.TIC } & E2. Adaptación & 1. Sí; 2. No \\
\hline & & E2. Compaginar & 1. Sí; 2. No \\
\hline & \multirow{2}{*}{$\begin{array}{l}\text { E3. Formación } \\
\text { permanente }\end{array}$} & E3. Necesidad de formación & 1. Sí; 2. No \\
\hline & & E3. Contenido de formación & Nominal-Cadena \\
\hline & E4. Retos & E4. Retos educación s. XXI & Nominal-Cadena \\
\hline & E5. Satisfacción & E5. Satisf. con la profesión & 1. Sí; 2. No \\
\hline
\end{tabular}

Fuente: elaboración propia

\section{Resultados}

\section{Factores de la identidad docente relacionados} con la infancia-adolescencia

Esta primera categoría se centra en conocer los microrrelatos personales, familiares y escolares que vivieron los docentes de educación parvularia durante su infancia y adolescencia, así como la relevancia que atribuyeron a estos en la determinación de su identidad docente. Los resultados de los datos generales de identificación personal muestran que el $95.1 \%$ de los participantes son mujeres de edad avanzada, de las cuales el $82.9 \%$ nacieron y vivieron su infancia en la región de Murcia, en un total de 37 municipios, siendo las ciudades de Murcia, Cartagena, Lorca, Águilas y Molina de Segura las más representadas, en ese orden indicado. Entre los docentes que nacieron en otras comunidades autonómicas, predomina la Comunidad de Valencia, concretamente Alicante, seguida de Madrid y Cataluña. 
Respecto a los factores familiares, se evidencia una escasa diversidad familiar, pues el $80.8 \%$ pertenecían a familias nucleares, mientras el $17.1 \%$, a familias extensas, siendo la monoparentalidad una excepción puntual en la que se encontraban tan solo 2 de los 123 docentes. Se trataba de unidades familiares numerosas, de las cuales el $64.5 \%$ de las familias estaba compuesta por más de 4 y menos de 11 miembros. El valor concedido a los hijos como aporte cultural-religioso y soporte para el trabajo familiar se hace patente en el escaso volumen de familias con hijos únicos ( $n=6$; $5.7 \%$ ), estableciéndose los valores promedio en 2 o 3 hijos por unidad familiar. No obstante, el 31.4 \% de las familias tenían más de 3 hijos, con un rango máximo de 8 hijos. En definitiva, la familia nuclear conformada por los progenitores y 2 o 3 hijos es el modelo familiar más predominante (30.8\% y $29.9 \%$, respectivamente).

En cuanto a los factores educativos, se observa que la subcategoría referida al estado de la educación y al tipo de escolaridad vivida durante la infancia y adolescencia comparten algunas unidades de información entre los entrevistados. De ahí que el análisis de contenido de ambas cuestiones se ha realizado conjuntamente. Tan solo cinco docentes consideran que la educación vivida durante su infancia fue similar a la actual, el resto señala diversas diferencias (en total 260), pues cada docente podía hacer referencia en su relato a varios rasgos de la escolaridad vivida.

En la tabla 2 se presentan los rasgos que caracterizan y definen la educación y escolaridad que recibieron los docentes. La mayoría de ellos hace mención a la normativa vigente del momento, es decir, al carácter religioso de la educación, que era altamente autoritaria y tradicional, separada por sexos.

Tabla 2

Rasgos característicos de la educación y escolaridad vivida por los docentes durante su infancia-adolescencia

\begin{tabular}{l|c|c|l|c|c}
\hline Educación/Escolaridad & $\mathbf{n}$ & $\mathbf{\%}$ & Educación/Escolaridad & $\mathbf{n}$ & $\mathbf{\%}$ \\
\hline Leyes educativas & 52 & 42.3 & Jornada partida & 8 & 6.5 \\
\hline Religioso & 34 & 27.6 & Comunitaria (familia-escuela) & 7 & 5.7 \\
\hline Autoritaria (distante y exigente) & 30 & 24.4 & No obligatoria & 6 & 4.9 \\
\hline Tradicional & 27 & 22 & Escasos recursos & 6 & 4.9 \\
\hline Separada por sexo & 21 & 17.1 & Valores & 4 & 3.2 \\
\hline Naturaleza del centro & 19 & 15.4 & Coser & 4 & 3.2 \\
\hline Metodología & 11 & 9 & Masificadas & 3 & 2.4 \\
\hline Unitaria & 10 & 8.1 & Otras & 8 & 6.5 \\
\hline Rural & 10 & 8.1 & & & \\
\hline
\end{tabular}

Fuente: elaboración propia 
Otro aspecto relevante en la escolarización es la relación que los encuestados mantenían con los miembros de la comunidad educativa (tabla 3). En este sentido, se registraron hasta tres unidades informativas para referirse a la relación con el profesorado, predominando: 1) la cercanoafectiva; 2) la autoritaria; 3) la distante, marcada por el respeto. Asimismo, las relaciones con sus compañeros eran buenas y de amistad. Finalmente, se identificaron las relaciones entre familia y escuela (86 \%) que, a pesar de ser mínimas y distantes, se mantenían focalizadas en las reuniones o tutorías. En contraposición, algunos docentes vivieron una escolaridad caracterizada por relaciones cercanas, frecuentes y comunitarias entre familia y profesorado.

Tabla 3

Relaciones con los profesores, compañeros y relación familia-escuela

\begin{tabular}{l|c|c|l|c|c|l|c|c}
\hline Profesorado & $\mathbf{n}$ & $\mathbf{\%}$ & Compañeros & $\mathbf{n}$ & $\mathbf{\%}$ & Familia-escuela & $\mathbf{n}$ & $\mathbf{\%}$ \\
\hline Afectivo-cercano & 78 & 63.4 & Amistad & 92 & 74.8 & Mínima-distante & 50 & 40.6 \\
\hline Autoritario & 45 & 36.6 & Negativas & 4 & 3.2 & Reuniones-tutoría & 29 & 23.6 \\
\hline Distante-respeto & 45 & 36.6 & Variable & 4 & 3.2 & Mucha-cercana & 22 & 17.9 \\
\hline Pedagógico & 14 & 11.4 & Normal & 2 & 1.6 & Comunitaria & 19 & 15.6 \\
\hline Tradicional & 10 & 8.1 & lgualdad & 1 & 0.8 & Tratar problemas & 13 & 10.6 \\
\hline & & & & & & Asociación de madres y & 5 & 4.1 \\
& & & & & & padres de alumnos (AMPA) & & \\
\hline & & & & & & Deberes & 2 & 1.6 \\
\hline & & & & & & Otras & 7 & 5.7 \\
\hline
\end{tabular}

Fuente: elaboración propia

Finalmente, el $78.9 \%$ de los docentes aprovecharon la oportunidad de comentar otros aspectos de este periodo, indicando que el centro educativo y el profesorado los marcaron positivamente $(n=44)$, como también el amor por los libros o las artes $(n=3)$; por el contrario, otros tuvieron experiencias negativas en su escolaridad, que impulsaron el deseo de querer cambiar esa situación ( $n=21$ ). Otros más señalaron la influencia de la familia en esta etapa ( $n=15)$, resaltando que algunos de sus parientes eran maestros $(n=4)$ o el duelo de perder un familiar cercano $(n=4)$.

\section{Factores relacionados con la toma de decisiones}

\section{de la docencia como profesión}

Las cuestiones contempladas en esta etapa permitieron reconocer los elementos que incidieron en la decisión de convertirse en docentes de educación infantil. Los breves relatos recabados señalan un momento concreto de sus vidas asociado a la infancia (57.4\%), adolescencia (29.5\%) o madurez (13.1\%). A pesar de que la decisión se tomó de forma autónoma (89.3\%), solo el 75.2 \% contó con el apoyo de la familia, mientras que el 
$11.3 \%$ estuvo motivado por algún docente y el $1.5 \%$ por los amigos; en algunos casos, los encuestados comenzaron a estudiar otra carrera y más tarde se cambiaron a educación infantil (9\%). La mayoría de los docentes comenzaron sus estudios universitarios entre 1971-1990 (48.8\%) o entre 1991-2010 (39.1\%).

\section{Factores de la identidad docente relacionados}

\section{con el paso por la universidad}

La formación inicial contribuyó con la capacitación de los docentes de educación infantil para su ejercicio profesional, de ahí que haya sido considerada como una etapa relevante en la construcción de la identidad docente. En esta categoría se pretende conocer la valoración docente de la titulación, el papel de los formadores y la aplicabilidad de los aprendizajes adquiridos.

En primer lugar, los relatos sobre el estado de los estudios universitarios de los encuestados comprenden una amplia horquilla temporal que aporta unidades de información variada e incluso contradictoria (tabla 4). La mayoría de los docentes estudió una diplomatura (31.7\%), y el $13.8 \%$ se formó en diversas especialidades, mientras que el 9.7 \% obtuvo el grado en educación infantil. No obstante, este tipo de formación ha tenido épocas en las que ha estado infravalorada (28 \%) o sin notas de corte $(9.7 \%)$, y otras en las que ha tenido mucha demanda (17.9\%), razón por la cual el ingreso a esta carrera requiere de notas de corte elevadas (3.2\%).

Tabla 4

Rasgos que caracterizan los estudios universitarios de educación infantil

\begin{tabular}{l|c|c|l|c|c}
\hline Educación/Escolaridad & $\mathbf{n}$ & $\mathbf{\%}$ & Educación/Escolaridad & $\mathbf{n}$ & $\mathbf{\%}$ \\
\hline Diplomatura & 39 & 31.7 & Grado & 12 & 9.7 \\
\hline Infravalorada & 28 & 22.8 & Prácticas en centros & 7 & 5.7 \\
\hline Muy teórica & 27 & 22 & Tradicional & 7 & 5.7 \\
\hline Muy demandada & 22 & 17.9 & Vocacional & 7 & 5.7 \\
\hline Diversas especialidades & 17 & 13.8 & Feminizada & 4 & 3.2 \\
\hline Sin nota de corte & 12 & 9.7 & Nota de corte alta & 4 & 3.2 \\
\hline
\end{tabular}

Fuente: elaboración propia

En lo que respecta al profesorado universitario que imparte clases en carreras de educación infantil, se definen principalmente por la transmisión de conocimiento (98.3\%), aunque también se muestran cercanos (66.4\%), especialmente los más jóvenes, y otros escasamente politizados (34.5\%). A pesar de su marcado papel tradicional, el $57.3 \%$ de los entrevistados 
consideran que entre sus profesores universitarios han encontrado a alguien que ha actuado como modelo educativo y les ha marcado en su labor docente, aunque también consideran que los profesores que han tenido en otros niveles no universitarios han resultado modelos más ajustados a su realidad profesional. Esto se corrobora al preguntarles por la aplicación de los aprendizajes en su praxis profesional, pues a pesar de que el $71.6 \%$ de los docentes de educación infantil reconoce haber utilizado lo aprendido, el $33.7 \%$ admite que poco o nada de estos conocimientos han sido aplicados, y el $21.3 \%$ solo lo ha hecho en alguna ocasión. Apenas 40 de los 123 docentes entrevistados considera que lo que han aprendido ha sido implementado con mucha o bastante frecuencia. Finalmente, tan solo un tercio de los entrevistados incluye en la valoración de la utilidad de los estudios universitarios elementos específicos del tipo de aprendizajes. La teoría ha sido calificada ambiguamente $(n=14)$, de forma positiva pero también de forma crítica, reconociendo que se olvida con el tiempo. Se consideran más útiles las practicas $(n=7)$ y las competencias personales $(n=7)$. Otros, además, recuerdan con cariño algunas asignaturas que les han aportado recursos para afrontar la praxis profesional $(n=11)$.

\section{Factores del ejercicio docente relacionados con la identidad}

Se trata de dimensiones más amplias que las contempladas en la entrevista, que abarcan aspectos como el reconocimiento docente, las relaciones con el profesorado y alumnado, el sentimiento de aislamiento, la infravaloración o discriminación de género, la estabilidad emocional, los cambios docentes y su transferencia al centro educativo.

Partimos de la consideración de que el reconocimiento social de cualquier profesión es un aspecto esencial, que incide en la probabilidad de su elección y en la satisfacción de su desempeño. Al respecto, el $70.8 \%$ de los docentes de educación básica entrevistados se han sentido reconocidos por las familias $(n=45)$, por la sociedad $(n=30)$, por los compañeros $(n=25)$ y por los niños $(n=7)$. De hecho, el $82.15 \%$ mantiene relaciones personales y profesionales satisfactorias con sus compañeros, y tan solo un $6.5 \%$ se ha sentido aislado en algún momento.

Como se observa en la tabla 5, las relaciones con el alumnado se describen muy satisfactorias, por el hecho de ayudar al niño a superarse y alcanzar sus logros (16.31\%). Las experiencias predominantemente afectivas, llenas de abrazos, muestras de cariño, buenos recuerdos, representan el $48.8 \%$. También son gratas las fiestas y convivencias que se comparten con los niños y sus familias (4.1\%). En comparación con lo anterior, a los docentes les cuesta más narrar sucesos negativos, de los cuales los comportamientos disruptivos y alborotadores son los más molestos (17.9\%), seguidos 
por los problemas con las familias (15.4\%) y los presentados durante el trabajo en contextos de vulnerabilidad (13.8\%), situación poco grata para los docentes.

Tabla 5

Experiencias positivas y negativas con el alumnado que marcan la identidad del docente de infantil

\begin{tabular}{l|c|c|l|c|c}
\hline Experiencias positivas & $\mathbf{n}$ & $\mathbf{\%}$ & Experiencias negativas & $\mathbf{n}$ & $\mathbf{\%}$ \\
\hline Afectivas & 60 & 48.8 & Ninguno & 21 & 17.2 \\
\hline Logros y superación en el niño & 20 & 16.3 & Disruptivas-violencia & 22 & 17.9 \\
\hline Fiestas y convivencias & 5 & 4.1 & Problemas con las familias & 19 & 15.4 \\
\hline Trabajar con niños con necesidades & 3 & 2.4 & Centro marginal o diversidad & 17 & 13.8 \\
\hline Ayudar a las familias & 3 & 2.4 & Accidentes o lesiones & 9 & 7.3 \\
\hline Muchos momentos (indefinido) & 9 & 7.3 & Frustración por no ayudarlos & 9 & 7.3 \\
\hline Otros & 6 & 4.9 & Inexperiencia-profesor novel & 8 & 6.5 \\
\hline & & & Emocionales & 7 & 5.7 \\
\hline
\end{tabular}

Fuente: elaboración propia

Otro aspecto de la praxis educativa que ha sido señalado por el $91.1 \%$ de los docentes entrevistados es la estabilidad emocional en el aula, ya que los niños son como esponjas, y por ello necesitan de positividad y equilibrio, para que no se afecte su aprendizaje (82.9\%). El contexto también incide en el bienestar emocional del alumnado (50.4\%), especialmente el familiar $(n=37)$, seguido del social, referido a los aspectos económicos, comunitarios y culturales $(n=16)$ y, finalmente, algunos profesores señalaron el propio contexto escolar $(n=4)$. Por otra parte, respecto al desgaste emocional asociado al desempeño profesional, la mayoría de los docentes admitieron no tener conocimiento al respecto (63.6\%); otros estaban al tanto, pero no imaginaban que fuera tan difícil desconectar las problemáticas del centro educacional, especialmente las referidas al alumnado, y unos pocos tenían conocimiento de ello, porque habían trabajado previamente con menores (36.4\%); todo esto contribuye a que el docente como tal vaya evolucionando (94.6\%).

Finalmente, el $35.3 \%$ de los docentes entrevistados se han sentido en alguna ocasión infravalorados, principalmente por las familias $(n=16)$, por otros profesores, generalmente de niveles superiores $(n=12)$, o por la sociedad $(n=3)$ y la administración $(n=2)$, aunque tratan de que esto no les afecte $(n=8)$. Con menor porcentaje se encuentran los docentes de educación infantil que se han sentido discriminados por su género (7.3 \%).

\section{Factores de la identidad docente relacionados con la madurez}

La última de las etapas se centra en los años de madurez, lo que nos permite identificar si se han vivido cambios de ley educativa durante los 
años de actividad laboral. Este es un aspecto relevante, ya que la finalidad de las políticas es la de introducir cambios en la práctica profesional, generando dilemas entre la docencia carismática y la burocrática, desafiando de este modo la identidad del docente (Carrasco et al., 2019; Sola et al., 2020). En esta situación se encuentran en $87.5 \%$ de los entrevistados, quienes consideran que los cambios de ley contribuyen a incrementar el papeleo burocrático (31.4\%), pero no inciden significativamente en el aula (32.4\%), a pesar de que sí afectan al currículo y a lo metodológico (16.3 \%), y suponen una oportunidad para el cambio y actualización (2.9\%). Los docentes denuncian el cambio continuo (5.9\%), a veces para peor (2.9\%), y reivindican el pacto educativo, no del tipo jerarquizado, sino contando con los docentes. Algunos de los cambios recientes que han incorporado las leyes educativas a los centros son el uso de las TIC y la educación emocional. Al respecto, los docentes entrevistados, independientemente de la edad, reconocen que se han adaptado adecuadamente a las TIC (87.5\%), empleando cursos para ello (28.4\%), y que logran compaginar bien esto con la enseñanza tradicional (74.3\%). El $41.5 \%$ de los docentes reconoce haber sido formado en educación emocional, aunque también se han registrado otras temáticas de formación (tabla 6). En general, los resultados evidencian que la formación permanente se ha consolidado como rasgo de identidad docente, ya que el $92.1 \%$ admite haber sido formado, y le otorgan una gran relevancia en sus relatos.

En el tema de los retos educativos para el siglo XXI se han recabado un total de 165 unidades de análisis muy dispares, que han sido agrupadas en las categorías que se muestran a continuación. Prevalecen los intereses curriculares, especialmente referidos a las pedagogías emergentes (32.5\%); otro gran reto que arrastramos de años atrás es la educación en valores, contemplando como hitos relevantes la construcción del ser, la ciudadanía y el medio ambiente, entre otros (24.6\%).

Tabla 6

Contenido de la formación permanente y retos educativos en el siglo XXI

\begin{tabular}{l|c|c|l|c|c}
\hline Formación permanente & $\mathbf{n}$ & $\mathbf{\%}$ & Retos educativos & $\mathbf{n}$ & $\mathbf{\%}$ \\
\hline Educación emocional & 51 & 41.5 & Curriculares-metodológicos & 40 & 32.5 \\
\hline TIC & 35 & 28.4 & Valores & 34 & 27.6 \\
\hline Metodológicos & 19 & 15.4 & TIC-recursos & 25 & 20.3 \\
\hline Disciplinas & 16 & 13.1 & Docente & 19 & 15.4 \\
\hline Valores & 4 & 3.2 & Política y calidad educativa & 16 & 13.1 \\
\hline Innovación & 3 & 2.4 & Inclusión-integración & 15 & 12.2 \\
\hline Otros & 1 & 0.8 & Emociones & 13 & 10.6 \\
\hline & & & Otros & 3 & 2.4 \\
\hline
\end{tabular}


Terminamos esta exposición señalando que casi la totalidad de los docentes de educación infantil se encuentran muy satisfechos con la elección profesional realizada (99.1\%), y tan solo una persona cambiaría a un nivel de docencia superior.

\section{Conclusiones y discusión}

Las entrevistas realizadas a más de un centenar de docentes de educación infantil, que han desarrollado su ejercicio en el contexto de la región de Murcia (España), permite tomar el pulso a una muestra en la que es palpable la feminización de esta profesión, algo que se advierte de forma clara y reiterada en las matrículas anuales en formación inicial docente para esta especialidad concreta. Es también reseñable el proceso de envejecimiento de las plantillas, en una sociedad envejecida como la española, con más de un $30 \%$ de participantes mayor de cincuenta años. Estas dos características coinciden, como no podía ser de otra forma, con las descripciones sociodemográficas que anualmente y por comunidades autónomas aborda el Ministerio de Educación, entre otras entidades, a través de los informes anuales de seguimiento del sistema educativo (Ministerio de Educación y Formación Profesional, 2019).

Algunos de los resultados relativos a factores de identidad docente relacionados con la infancia-adolescencia nos aproximan a una realidad en la que, a esta feminización, se une un cierto sedentarismo en las profesionales de este ámbito, en tanto que las profesionales de la educación infantil se emplazan y ejercen la profesión en su propia región (82.9\%), y los municipios más grandes y poblados son también los más representados en el aspecto de procedencia de las docentes. El factor de inmigración laboral, en lo que a la profesión de docencia infantil se refiere, aun siendo minoritario, se concentra en zonas cercanas geográficamente, prioritariamente Alicante, o bien en procedencias de grandes núcleos urbanos, como Madrid y Cataluña.

La procedencia familiar para las docentes es también significativamente superior desde familias nucleares, lo que da indicios de su carácter de modelo familiar ampliamente asentado en España, y aún es muy escasa, prácticamente marginal, la representación de la monoparentalidad en este aspecto.

En las descripciones de su propia escolaridad, es común la referencia a los niveles educativos asociados a la legislación que los ordena (Educación General Básica para la Ley General de Educación, 1970; Enseñanza Secundaria Obligatoria para la LOGSE, 1990; etc.), la titularidad de los centros como factor distintivo, y que expresan reiteradamente aquellas maestras 
que proceden de centros de enseñanza religiosa o concertada, y elementos vinculados a figuras y medidas de autoridad o su ausencia, lo que sigue haciendo ostensible un imaginario de control social asociado a la institución docente y a las funciones que debe cumplir en el seno de las sociedades, se expliciten o no.

En el recuento de relaciones vividas durante la infancia y adolescencia, la cercanía y afectividad con el profesorado se imponen a la consideración autoritaria. Se señala también el respeto al docente, como rasgo característico de la narrativa biográfica que describe su paso por los niveles educativos obligatorios. Hacerlo expreso significa incurrir, en muchos de los casos, en apreciaciones simples comparativas, en las que se resalta dicho carácter en contraposición a la situación actual, donde el respeto se vive como reto de la educación por venir. En ese sentido, Escudero (2019) se refiere explícitamente a la necesidad de conectar ambas experiencias: "Tan importante es idear y comprometer mejoras y transformaciones valiosas y necesarias de cara al futuro como, al mismo tiempo, conectarlas reflexiva y críticamente con la historia previa [...] y con las regularidades del presente" (p. 168).

El componente emocional en la construcción de la identidad es reconocido en diversos estudios (Buitrago y Cárdenas, 2017; Monereo y Domínguez, 2014) y ha sido manifestado por los participantes en varios momentos de sus itinerarios vitales. La mayoría valora como buenas y de amistad las relaciones con sus compañeros, aunque existe también un porcentaje que detalla en primera persona casos de acoso escolar, caracterizándolos como propios de las instituciones docentes y de la vida escolar, y no específicos de la época actual.

En lo que respecta a las relaciones entre familia y escuela se indica su presencia, y también su carácter puntual y mínimo de forma mayoritaria, que contrasta con la percepción de cambio acaecido en el contexto escolar, lo que nos permite coincidir con Tejada (2018), cuando se refiere a la tensión existente entre una visión nostálgica del pasado de la profesión y un presente en crisis. Un buen número de docentes señala sus vivencias con el profesorado y su paso por el centro educativo en las etapas de infancia y adolescencia como una influencia positiva en sus vidas.

Si hacemos uso de las dimensiones trabajadas por Monereo y Domínguez (2014), podemos tomar conciencia de que los elementos relacionados con la toma de decisiones de la docencia como profesión vuelve a aparecer un factor básicamente vocacional, que se toma en la mayoría de los casos de forma prematura (57.64 \% en la infancia y $29.5 \%$ en la adolescencia). La percepción de los docentes es la de una decisión tomada de forma autónoma, de la que se hacen responsables, en un porcentaje elevado de los casos contando con apoyo familiar, y que coincide con una elevada 
satisfacción con dicha decisión, lo que, según otros estudios (Cantón et al., 2017; González y Subaldo, 2015), se mantiene entre los futuros docentes, incluso aunque las condiciones laborales mermen en sueldo, funciones y evaluaciones de su trabajo. La mitad de los encuestados estudió su carrera antes de los años noventa, y casi un $40 \%$ hasta el 2010, lo que hace que la representatividad de los graduados superiores sobre los diplomados universitarios sea aún minoritaria en el ejercicio docente (9.7\%).

Las vivencias de formación inicial docente hacen manifiesta la evolución de la profesión en el contexto nacional, pues la variedad de edades de las maestras permite tomar conciencia del desarrollo producido desde unos estudios infravalorados, sin nota de corte y en los que la selectividad no era requisito, a estudios con plena entidad de formación universitaria superior y en los que el carácter selectivo los convierte en altamente demandados (Ayala y Luzón, 2013). La dispersión de resultados permite en cualquier caso una tipificación de enseñanza eminentemente teórica para los estudios anteriores a 1990 y una valoración expresa de las prácticas en fechas más recientes. La visión mayoritaria del docente universitario como transmisor de conocimiento no exime del uso ni de la repercusión de la formación recibida para su práctica laboral en más del 70 \% de las maestras encuestadas, aunque con niveles de aplicación baja.

Los factores del ejercicio docente relacionados con la identidad y analizados aglutinan una serie de factores relativos a emociones, relaciones y sentimientos de aislamiento. El desenvolvimiento social juega también un papel significativo en la construcción de la propia identidad, y alcanza un $82.15 \%$ con buenas relaciones con sus compañeros. En relación a consideraciones a tener en cuenta para la carrera docente, Fernández-Enguita (2019) se refiere a "evitar ignorar que se trata de una actividad en la que el físico (voz, columna vertebral), el ego (siempre en público, aunque sea un público cautivo) y la disposición (tratar con menores, etc.) están sujetos a altos niveles de exposición y riesgo" (p. 209). Un alto reconocimiento social autopercibido de los participantes deja sobre la mesa que un $35 \%$ de las y los docentes han sido infravalorados por familias y profesores de otros niveles. La inseguridad de los noveles ha sido puesta de manifiesto como un aspecto negativo en la interacción con el alumnado. No solamente está presente el miedo escénico, sino también el sentimiento de inseguridad, al considerarse menos que el resto de sus compañeros con más experiencia docente (Correa, 2014). Las voces de las maestras nos dicen de forma contundente que la percepción de discriminación por género es baja y minoritaria (7.3\%) en la profesión.

En el marco de lo que Monereo y Domínguez (2014) consideran como representaciones sobre los procesos instruccionales, de enseñanza, 
aprendizaje y evaluación, se inscriben de forma más clara los factores que se relacionan con la madurez del ejercicio docente. Más del $80 \%$ ha vivido cambios legislativos, lo que no hace sino dejar patente el vaivén de reformas educativas en España ampliamente referenciadas. De ese vaivén, y su vínculo al papeleo burocrático que se asocia a la profesión, la representatividad del $31.4 \%$ hallada estaría en consonancia con las apreciaciones de Tardif y Le Vasseur (2018) sobre los docentes en los países occidentales, ya que la mayoría son "funcionarios del Estado: en este contexto, estarían revestidos de una misión que les confieren las autoridades públicas y educativas, y su identidad estaría modelada en gran parte por las numerosas normas y reglas propias de su función" (p. 20).

Otro componente o dimensión de la auto representación del profesor en relación con su identidad docente (Monereo y Domínguez, 2014) se concentra en las representaciones sobre el propio rol profesional (especialista, docente y tutor, investigador, gestor, profesional). En este marco, según los resultados del ejercicio, un $16.31 \%$ tiene que ver con el objeto de la educación, la superación y el alcance de logros, y la mayoría son recuerdos de corte afectivo (60\%). Los comportamientos disruptivos, los problemas con las familias y el trabajo en contextos de vulnerabilidad son los relatos más negativos, asociados a las dificultades encontradas en el desempeño profesional, que curiosamente no se hacen presentes en los retos a afrontar por la educación en años venideros.

Las profesoras Pardo y Adlerstein (2016) se refieren a la complejidad de atender esta diversidad creciente, en la medida en que "es cada vez mayor la literatura especializada y la investigación comparada que muestran cómo estas profesionales deben enfrentar el complejo desafío de atender y enseñar a infancias diversas" (p. 136). También, la formación permanente se consolida como rasgo de identidad docente, y el 92.1 \% reconoce esa formación como necesaria en la línea de la caracterización del docente como un profesional reflexivo (Saiz-Linares et al., 2019), con una incidencia alta de formación tecnológica, metodológica, en general, y en emociones, que concentra prácticamente los tópicos de formación señalados. Esta formación continua, no ajustada a los puntos en los que han encontrado dificultades, coincide con los hallazgos de Tejada (2018), cuando refiere que a pesar de la amplia oferta formativa existente siguen siendo necesarios planes específicos, ligados a las necesidades de mejora, y a la aportación de Marcelo y Gallego (2018), quienes afirman que no se trata de hacer al docente más experto, sino de "mantener la actualización que requieren sus capacidades" (p. 50).

En relación a los retos que afronta la profesión docente de acuerdo a las opiniones de las entrevistadas, los mismos deben ser considerados en consonancia con la percepción de cambio necesario: 
El saber, se dice ahora, está socialmente y profesionalmente distribuido, y ello debiera tomarse como una buena invitación a tomar la nota debida de las percepciones, juicios, vivencias, condiciones laborales, sueños y frustraciones que los sujetos más directamente afectados viven y sienten y esperan de los cambios que podrían y deberían ocurrir. (Escudero, 2019, p. 170)

En esta línea, y en un abanico amplio y dispar, los retos curriculares y metodológicos son los que concentran el interés mayoritario de los profesionales, a los que se unen consideraciones teóricas y filosóficas sobre la búsqueda del sentido de formación de personas en el trabajo que se desempeña.

En lo referente al proceso de investigación, existen limitaciones vinculadas, por un lado, a la extensión de la entrevista, lo que ha derivado en narrativas segmentadas, perdiendo algunos matices del relato único, y por otro, al volumen de la muestra, lo que garantiza una mayor riqueza de subjetividades, pero dificulta la categorización triangulada. No obstante, este estudio proporciona un amplio conocimiento de las atribuciones que los docentes de educación infantil tienen respecto a su identidad y posibilita la creación de cuestionarios, aunque su verdadera riqueza radica en la cantidad de interrogantes que genera. A continuación, a modo de ejemplo, formulamos, entre otros, los siguientes: ¿Cómo contribuir desde la universidad a una identidad docente más reflexiva, más activa y dialógica? ¿Qué diferencias existen en la identidad del docente en función de la etapa educativa? ¿Cómo cambia la identidad con el transcurso del tiempo? ¿Qué matices presenta la identidad del docente masculino de infantil?

En conclusión, no cabe duda entonces que aún queda un largo camino por recorrer en la investigación sobre la identidad del docente de educación infantil.

\section{Aclaraciones y agradecimientos}

Se asumió este estudio con el presupuesto de los equipos de investigación y a partir de ahí se desarrolló colaborativamente la realización de las entrevistas. De hecho, se tiene la intención de seguir profundizando en estos temas de gran relevancia, tanto para los docentes en ejercicio, como para los futuros profesionales. También es posible recabar información acerca de la percepción de los docentes sobre su proceso académico en la universidad.

\section{Sobre las autoras}

María Ángeles Hernández-Prados es licenciada y doctora en Pedagogía. Profesora titular de la Universidad de Murcia, España. Imparte docencia en el Departamento de Teoría e Historia de la Educación y desarrolla las siguientes líneas 
de investigación: Educación para la ciudadanía y mejora de la convivencia escolar, Educación en valores en el contexto familiar y Perspectiva ético-moral de la sociedad de la información, así como la relación entre familia y centros escolares.

Amalia Ayala De la Peña es profesora titular de Educación Comparada en el Departamento de Teoría e Historia de la Educación de la Facultad de Educación de la Universidad de Murcia, España. Doctora en Educación y doctora europea por la Universidad de Murcia, España. Es miembro del grupo de investigación: Inclusión social, educación y territorio. Sus últimas investigaciones se centran en políticas de formación del profesorado, inclusión educativa, espacio y tiempo en educación, infancia y educación, y administración de políticas escolares en perspectiva internacional.

\section{Referencias}

Ayala, A. y Luzón, A. (2013). Presentación: Retos y desafíos de la formación del profesorado en el siglo XXI. Una visión comparada. Revista Española de Educación Comparada, 22, 9-17. https://doi.org/10.5944/reec.22.2013.9320

Balderas-Gutiérrez, I. (2013). Propuesta de guion de entrevista para el estudio de la identidad docente. Revista Latinoamericana de Metodología de la Investigación Social, 3(6), 73-87. https://dialnet.unirioja.es/servlet/ articulo?codigo $=5275923$

Blasco-Hernández, T. y Otero-García, L. (2008). Técnicas conversacionales para la recogida de datos en investigación cualitativa: la entrevista (I). Nure Investigación, 33. https://www.nureinvestigacion.es/OJS/index.php/nure/article/ view/408/399

Bolívar, A. (2007). O esforço reflexivo de fazer da vida una historia. Pátio, 43, 12-15.

Bolívar, A. (2014). Las historias de vida del profesorado: voces y contextos. Revista Mexicana de Investigación Educativa, 19(62), 711-734. http://www.scielo. org. $\mathrm{mx} /$ scielo.php?script=sci_arttext\&pid=S1405-66662014000300004

Buitrago, R. y Cárdenas, R. N. (2017). Emociones e identidad profesional docente: relaciones e incidencia. Praxis y Saber, 8(17), 225-247. https://doi.org/ 10.19053/22160159.v8.n17.2018.7208

Cantón, I., García-Martín, S. y Cañón, R. (2017). Construcción de la identidad profesional en los futuros maestros de infantil. Revista Infancia, Educación y Aprendizaje, 3(2), 612-617. https://doi.org/10.22370/ieya.2017.3.2.789

Cantón-Mayo, I. y Tardif, M. (eds.) (2018). Identidad profesional docente. Narcea.

Carbonero, M. Á., Reoyo, N., Freitas, A. y Valdivieso, J. A. (2012). Perfil del profesor de infantil: valoración cualitativa. International Journal of Developmental and Educational Psychology, 2(1), 417-426. http://infad.eu/RevistalNFAD/in dex.php/publicaciones/revista-infad-2012/revista-infad-2012-no1-volumen2-pp-417-426/

Carrasco, C., Luzón, A. y López, V. (2019). Identidad docente y políticas de accountability: el caso de Chile. Estudios Pedagógicos, 45(2), 121-139. http:// dx.doi.org/10.4067/S0718-07052019000200121 
Carrasco, V., Hernández, M. J. e Iglesias, M. J. (2012). Aportaciones de los maestros en formación a la construcción del perfil del docente competente desde la reflexión en el aula. Teoría de la Educación. Educación y Cultura en la Sociedad de la Información, 13(3), 290-316. https://doi.org/10.14201/eks.9142

Cisterna, F. (2005). Categorización y triangulación como procesos de validación del conocimiento en investigación cualitativa. Theoria, 14(1), 61-71. http:// www.redalyc.org/articulo.oa?id=29900107

Comisión Europea/EACEA/Eurydice (2019). Cifras clave de la educación y atención a la primera infancia en Europa- Edición 2019. Informe de Eurydice. Oficina de Publicaciones de la Unión Europea.

Correa, J. M. (2014). Empezar a trabajar en la educación infantil: las condiciones de acceso a la docencia en los relatos biográficos de las maestras principiantes Tendencias Pedagógicas, 24, 41-53. https://revistas.uam.es/tendencias pedagogicas/article/view/2091/2189

Escudero-Muñoz, J. M. (2019). Las profesión y formación docente en discusión: ¿cuál es el estado de la cuestión, qué cambiar y por qué, cómo habría de hacerse? En J. Manso y J. Moya (coords.), Profesión y profesionalidad docente. Una acción educativa comprometida con el desarrollo humano (pp. 167-176). ANELE-REDE.

Fernández-Enguita, M. (2019). Robustecer, vigorizar y revalorizar la profesión. En J. Manso y J. Mayo (coords.), Profesión y profesionalidad docente. Una acción educativa comprometida con el desarrollo humano (pp. 201-209). ANELE-REDE.

Gajardo-Asbún, K. P. (2019). Estado del arte sobre identidad docente: investigación de experiencias de profesores en formación y en ejercicio. IE Revista de Investigación Educativa de la REDIECH, 10(18), 79-93. https://dx.doi. org/10.33010/ie_rie_rediech.v10i18.217

González, J. y Subaldo, L. (2015). Opiniones sobre el desempeño docente y sus repercusiones en la satisfacción profesional y personal de los profesores. Educación, 47(24), 90-114. http://revistas.pucp.edu.pe/index. php/educa cion/article/view/14247

González, M., Muñoz, M., Cruz, A. y Olivares, M. A. (2019). Construcción de la identidad profesional docente en educación infantil en Córdoba (España). Revista de Ciencias Sociales, 25(3), 30-41. https://www.redalyc.org/jatsRe po/280/28060161002/index.html

Hurtado, J. (2010). Metodología de la investigación. Guía para la comprensión holística de la ciencia. Ediciones Quirón

Llorent, V. (2013). La educación infantil en Alemania, España, Francia e Inglaterra: estudio comparado. Revista Española de Educación Comparada, 21, 29-58. https://doi.org/10.5944/reec. 21.2013.7614

Marcelo-García, C. y Gallego-Domínguez, C. (2018). ¿Quién soy yo como maestro? Construcción de la identidad profesional en docentes principiantes. En I. Cantón-Mayo y M. Tardif (eds.), Identidad profesional docente (pp. 45-56). Narcea.

Ministerio de Educación y Formación Profesional (2019). Informe 2019 sobre el estado del sistema educativo. Centro de Publicaciones del Ministerio. https://www.educacionyfp.gob.es/dam/jcr:4f35ae94-f996-4ceb-b3f021b2e421ec26/i19cee-informe.pdf 
Monereo, C. y Domínguez, C. (2014). La identidad docente de los profesores universitarios competentes. Educación XX1, 17(2), 83-104. https://doi. org/10.5944/educxx1.17.2.11480

Norman, T. A. (2020). Methods of a Narrative Inquirist: Storying the Endured Teacher Identity. The Qualitative Report, 25(11), 3962-3975. https://nsuworks. nova.edu/tqr/vol25/iss11/10

OECD (2017). Starting Strong 2017: Key OECD Indicators on Early Childhood Education and Care. OECD Publishing. https://doi.org/10.1787/9789264276116-en.

Pardo, M. y Adlerstein, C. (2016). Estado del arte y criterios orientadores para la elaboración de políticas de formación y desarrollo profesional de docentes de primera infancia en América Latina y el Caribe. UNESCO Publishing.

Pereira-Pérez, Z. (2011). Los diseños de método mixto en la investigación en educación: Una experiencia concreta. Revista Electrónica Educare, 15(1), 15-29. https://doi.org/10.15359/ree.15-1.2

Pérez-Abril, M., Roa-Casas, C., Vargas, Á.P. e Isaza, L. A. (2014). ¿Qué caracteriza a un docente destacado? Rasgos de la práctica en los primeros grados de la escolaridad. Revista Colombiana de Educación, (67), 171-200. https://doi. org/10.17227/0120391.67rce171-200

Saiz-Linares, Á., Ceballos-López, N. y Susinos-Rada, T. (2019). Voz del alumnado y mejora docente. Una investigación en centros educativos en Cantabria. Revista Complutense de Educación, 30(3), 713-728. https://doi.org/10.5209/ rced.58883

Sarceda, M. C. (2017). La construcción de la identidad docente en educación infantil. Tendencias Pedagógicas, 30, 281-300. https://doi.org/10.15366/ tp2017.30.016

Sola, J., Sádaba, I. y Menéndez, C. (2020). Entre la docencia carismática y la burocratización de la enseñanza: las crisis de la sociología y los dilemas de su aprendizaje. RES. Revista Española de Sociología, 29(2), 419-427. https:// doi.org/10.22325/fes/res.2020.25

Tardif, M. y Le Vasseur, L. (2018). Los profesores en Canadá. ¿Una identidad profesional en mutación? En I. Cantón y M. Tardif (coords.), Identidad profesional docente (pp. 19-32). Narcea.

Tejada-Fernández, J. (2018). La búsqueda de la identidad laboral del profesorado. En I. Cantón y M. Tardif (coords.), Identidad profesional docente (pp. 75-94). Narcea. 